\title{
A simple implementation of the dual boundary element method using the tangential differential operator for plane problems
}

\author{
L. Palermo, P. C. Gonçalves \& L. G. Figueiredo \\ Faculty of Civil Engineering, Architecture and Urban Design, \\ State University of Campinas, Brazil
}

\begin{abstract}
Plane problems of linear fracture mechanics were solved with the dual boundary element method (DBEM) using the tangential differential operator (TDO) in traction boundary integral equation (BIE). The numerical implementation employed same shape functions for conformal and non-conformal interpolations with nodal parameters fixed at the ends of elements. Different collocation point positions were used in crack surfaces according to continuity requirements related to each BIE type employed. The aim of this paper is presenting a simple implementation for fracture analysis. Results obtained in the literature for stress intensity factors were included to show the accuracy of the formulation.
\end{abstract}

Keywords: tangential differential operator, dual boundary element method, stress intensity factor.

\section{Introduction}

This study began when the implementation presented in [1] was revisited in [2] with purpose to apply conformal interpolations along crack surfaces. Nonconformal interpolations along crack surfaces were used in [1] with quadratic elements and collocation points positioned at element nodes. The problems solved in [1] could be analyzed in [2] using conformal interpolations along crack surfaces with collocations points shifted to the interior of elements. The obtained results in [2] with quadratic or linear elements had a good agreement with those presented in [1] for internal positions ( $\left.\xi^{\prime}\right)$ of collocation points equal \pm 0.67 in the range $(-1,1)$. Furthermore, results were not significantly changed if conformal or 
non-conformal interpolation were applied along crack surfaces because their shape functions employed same expressions and nodal parameters were always fixed at the ends of elements. Traction BIE using the tangential differential operator (TDO) was studied in [3] with shape functions and collocation point positions studied in [2]. The order of the strong singularity was reduced with the operator and the displacement function derivative was adopted in the kernel containing TDO. Furthermore, an additional term related to TDO was introduced to allow discontinuities in the boundary line usually due to non-conformal interpolations at crack tips and corners when conformal interpolations were applied along crack surfaces. The results were not degenerated in [3] with reference to [1] or [2] even using conformal interpolations along crack surfaces and isoparametric linear elements.

Internal positions for collocation points were used in [1-3] for both BIEs of DBEM and the present study analyzes if collocation points of both BIEs should have same positions in opposite elements at crack surfaces when conformal interpolations are adopted or different positions could be applied.

\section{The dual boundary element method using the tangential differential operator}

The BIE for the gradient at an internal point $\mathrm{x}$ is written as follows using the differentiation in terms of field variables:

$$
u_{i, m}(x)=\int_{\Gamma} T_{i j, m}(x, y) u_{j}(y) d \Gamma(y)-\int_{\Gamma} U_{i j, m}(x, y) t_{j}(y) d \Gamma(y)
$$

$\mathrm{U}_{\mathrm{ij}}(\mathrm{x}, \mathrm{y})$ and $\mathrm{T}_{\mathrm{ij}}(\mathrm{x}, \mathrm{y})$ are the displacement and the traction, respectively, in direction $\mathrm{j}$ at the boundary point $\mathrm{y}$, due to a singular load in direction $\mathrm{i}$ at collocation point $\mathrm{x}$ ', according to the Kelvin solution for two-dimensional problems; $\mathrm{u}_{\mathrm{j}}(\mathrm{y})$ and $\mathrm{t}_{\mathrm{j}}(\mathrm{y})$ are the displacement and the traction at the field point, respectively.

After some algebraic manipulation and employing properties of Kelvin type fundamental solutions, the TDO is obtained from integration by parts $[3,4]$ and the BIE for the gradient at an internal point $\mathrm{x}$ with TDO is given by:

$$
u_{i, m}(x)=\int_{\Gamma} \sigma_{i b j}(x, y) D_{m b}\left[u_{j}(y)\right] d \Gamma(y)-\int_{\Gamma} U_{i j, m}(x, y) t_{j}(y) d \Gamma(y)
$$

$D_{b m}()$ is the tangential differential operator, which has the following definition:

$$
D_{m b}\left\lfloor u_{j}(y)\right\rfloor=n_{m}(y) u_{j, b}(y)-n_{b}(y) u_{j, m}(y)
$$

The integrals of eq. (2) are regular for internal points and exhibit singularities of order $1 / \mathrm{r}$ when the field point approaches the collocation point. Stress BIE is obtained from eq. (1) or eq. (2) using the Hooke tensor and the symmetry property of $\mathrm{U}_{\mathrm{ij}, \mathrm{m}}(\mathrm{x}, \mathrm{y})$. The limiting form of stress BIE at an internal point when 
it is led to a point on the boundary defines stress BIE at a boundary point, which has the following expression at point $\mathrm{x}$ ' on a smooth boundary:

$$
\begin{array}{r}
\frac{1}{2} \sigma_{\mathrm{ak}}\left(\mathrm{x}^{\prime}\right)=\mathrm{C}_{\mathrm{aki} \mathrm{m}} \int_{\Gamma} \mathrm{T}_{\mathrm{ij}, \mathrm{m}}\left(\mathrm{x}^{\prime}, \mathrm{y}\right) \mathrm{u}_{\mathrm{j}}(\mathrm{y}) \mathrm{d} \Gamma(\mathrm{y})-\int_{\Gamma} \sigma_{\mathrm{jak}}\left(\mathrm{x}^{\prime}, \mathrm{y}\right) \mathrm{t}_{\mathrm{j}}(\mathrm{y}) \mathrm{d} \Gamma(\mathrm{y}) \\
\frac{1}{2} \sigma_{\mathrm{ak}}\left(\mathrm{x}^{\prime}\right)=\mathrm{C}_{\mathrm{aki} m} \int_{\Gamma} \sigma_{\mathrm{ibj}}\left(\mathrm{x}^{\prime}, \mathrm{y}\right) \mathrm{D}_{\mathrm{mb}}\left[\mathrm{u}_{\mathrm{j}}(\mathrm{y})\right] \mathrm{d} \Gamma(\mathrm{y})-\int_{\Gamma} \sigma_{\mathrm{jak}}\left(\mathrm{x}^{\prime}, \mathrm{y}\right) \mathrm{t}_{\mathrm{j}}(\mathrm{y}) \mathrm{d} \Gamma(\mathrm{y})(4) \\
\mathrm{C}_{\mathrm{akim}}=\mu\left(\frac{2 v}{1-2 v} \delta_{\mathrm{ak}} \delta_{\mathrm{im}}+\delta_{\mathrm{ai}} \delta_{\mathrm{km}}+\delta_{\mathrm{am}} \delta_{\mathrm{ki}}\right)
\end{array}
$$

$\mathrm{C}_{\text {akim }}$ is the Hooke tensor for isotropic media, $v$ is the Poisson ratio and $\mu$ is equal to the shear modulus. $\delta_{\mathrm{ij}}$ is the Kronecker delta

An additional term is necessary in equation (4) when a discontinuity in the boundary line is introduced due to a displacement discontinuity:

$$
\begin{aligned}
\frac{1}{2} \sigma_{a k}\left(x^{\prime}\right) & =C_{a k i m} \int_{\Gamma} \sigma_{i b j}\left(x^{\prime}, y\right) D_{m b}\left[u_{j}(y)\right] d \Gamma(y)+ \\
& +\left[e_{3 b m} \sigma_{i b j}\left(x^{\prime}, y\right) u_{j}(y)\right]_{0}^{\Gamma}-\int_{\Gamma} \sigma_{j a k}\left(x^{\prime}, y\right) t_{j}(y) d \Gamma(y)
\end{aligned}
$$

$\mathrm{e}_{3 \mathrm{bm}}$ is the permutation symbol.

Traction BIE is obtained from eq. (3) or (5) when the stress tensor at boundary point $x^{\prime}$ is multiplied by direction cosines of the outward normal at this point $\left(\mathrm{n}_{\mathrm{a}}^{\prime}\right)$. The corresponding traction BIEs from eq. (3) and (5) are given by:

$$
\begin{aligned}
\frac{1}{2} \mathrm{t}_{\mathrm{k}}\left(\mathrm{x}^{\prime}\right)=\mathrm{n}_{\mathrm{a}}^{\prime}\left(\mathrm{x}^{\prime}\right) \mathrm{C}_{\mathrm{aki} \mathrm{m}} \int_{\Gamma} \mathrm{T}_{\mathrm{ij}, \mathrm{m}} \mathrm{u}_{\mathrm{j}} \mathrm{d} \Gamma-\mathrm{n}_{\mathrm{a}}^{\prime}\left(\mathrm{x}^{\prime}\right) \int_{\Gamma} \sigma_{\mathrm{jak}} \mathrm{t} \text { j } \mathrm{d} \Gamma \\
\frac{1}{2} \mathrm{t}_{\mathrm{k}}\left(\mathrm{x}^{\prime}\right)=\mathrm{n}_{\mathrm{a}}^{\prime}\left(\mathrm{x}^{\prime}\right) \mathrm{C}_{\mathrm{aki} \mathrm{m}} \int_{\Gamma} \sigma_{\mathrm{ibj}} \mathrm{D}_{\mathrm{mb}}\left(\mathrm{u}_{\mathrm{j}}\right) \mathrm{d} \Gamma+ \\
+\mathrm{n}_{\mathrm{a}}^{\prime}\left(\mathrm{x}^{\prime}\right)\left[\mathrm{e}_{3 \mathrm{bm}} \sigma_{\mathrm{ibj}} \mathrm{u}_{\mathrm{j}}\right]_{0}^{\Gamma}-\mathrm{n}_{\mathrm{a}}^{\prime}\left(\mathrm{x}^{\prime}\right) \int_{\Gamma} \sigma_{\mathrm{jak}} \mathrm{t}_{\mathrm{j}} \mathrm{d} \Gamma
\end{aligned}
$$

General mixed-mode crack problems can be solved with a single domain formulation in DBEM when the traction BIE (eq. (6) or (7)) is applied to one of the crack surfaces and the displacement BIE to the other. Although the integration path is still the same for coincident points on the crack surfaces, the respective boundary integral equations are now distinct. The displacement BIE is given by:

$$
\frac{1}{2} u_{i}\left(x^{\prime}\right)+\int_{\Gamma} T_{i j}\left(x^{\prime}, y\right) u_{j}(y) d \Gamma(y)=\int_{\Gamma} U_{i j}\left(x^{\prime}, y\right) t_{j}(y) d \Gamma(y)
$$

The collocation points must be positioned to satisfy the continuity requirements for each BIE. Continuity of the displacement function at $\mathrm{x}$ ' is the necessary condition for displacement BIE and it is satisfied when the collocation point is placed at the ends of the boundary element or at its interior. Continuity of the displacement derivative at $\mathrm{x}^{\prime}$ is required for traction BIE and it is satisfied when the collocation point is placed at the interior of the element $[1,4]$. 


\section{Numerical implementation}

The integrals of eq. (6) exhibit singularities of order $1 / \mathrm{r}$ and $1 / \mathrm{r}^{2}$. The strong singularity $\left(1 / \mathrm{r}^{2}\right)$ requires the use of the Cauchy and the Hadamard principalvalue integral. The application of second order finite part [1] results in analytical expressions for singular terms and a numerical integration using the GaussLegendre scheme for the regular term. Hadamard principal value integral is not used when traction BIE employs TDO because integrals of eq. (7) exhibit singularity types of order $1 / \mathrm{r}$ like in eq. (8). The improper integral only requires the use of the Cauchy principal value sense. Analytical expressions can be used to evaluate singular integrals with the Cauchy principal value sense and GaussLegendre scheme used for regular integrals. The present numerical implementation for terms containing the tangential differential operator considered the differentiation of the displacement function [5] and the following relation was applied:

$$
D_{b m}[f(y)]=n_{b}(y) f_{, m}(y)-n_{m}(y) f_{, b}(y)=e_{3 \gamma \rho} \frac{1}{|J|} \frac{d f}{d \xi}
$$

$\mathrm{J}$ is Jacobian of the transformation and $\xi$ is the intrinsic coordinate to perform the integration on the element; $\mathrm{f}$ corresponds to the function representing displacement in elements.

Linear shape functions were used to represent displacements and tractions in boundary elements. Same shape functions were used for conformal and nonconformal interpolations with nodal parameters positioned at ends of elements.

Collocation points for displacement BIE were positioned at nodes of elements in conformal interpolation and shifted to the interior of the element in nonconformal interpolation with positions $\left(\xi^{\prime}\right)$ equal \pm 0.67 in the range $(-1,1)$. On the other hand, collocation points for traction BIE were always shifted to the interior of elements and the positions $\left(\xi^{\prime}\right)$, in range $(-1,1)$, were: i) $\xi^{\prime}=-0.67$ for continuous elements; ii) $\xi^{\prime}=-0.67$ and $\xi^{\prime}=+0.67$ for discontinuous elements.

The diagonal terms were directly obtained using the shape function and the collocation point position in the element.

\section{Stress intensity factor evaluation}

Stress intensity factors were assessed using the near-tip displacement extrapolation [1]. Considering a polar coordinate system $(r, \theta)$ centered at the crack tip, such that crack surfaces could be defined with $\theta= \pm \pi$. The displacement field on the crack surface has the following expressions considering the first term of William's expansion:

$$
\begin{aligned}
& u_{2}(\theta=\pi)-u_{2}(\theta=-\pi)=\frac{\kappa+1}{\mu} K_{I} \sqrt{\frac{r}{2 \pi}} \\
& u_{1}(\theta=\pi)-u_{1}(\theta=-\pi)=\frac{\kappa+1}{\mu} K_{I I} \sqrt{\frac{r}{2 \pi}}
\end{aligned}
$$


The stress intensity factors for deformation modes I and II are $\mathrm{K}_{\mathrm{I}}$ and $\mathrm{K}_{\mathrm{II}}$, respectively; the parameter $\kappa$ is equal to $3-4 \eta ; \eta$ is equal to $v$ for plane strain problems and equal to $v /(1+v)$ for plane stress problems The near-tip displacement extrapolation works with eq. (9) and (10) to obtain stress intensity factors when the displacements are known. The situation is shown in Figure 1, where opposite elements share the crack tip at nodes B and C. The length of the linear element is equal to 1 . The stress intensity factors are given by:

$$
\begin{aligned}
& K_{I}^{D E}=\left(u_{2}^{D}-u_{2}^{E}\right) \cdot \frac{\mu}{\kappa+1} \cdot \sqrt{2} \cdot \sqrt{\frac{\pi}{l}} \\
& K_{I I}^{D E}=\left(u_{1}^{D}-u_{1}^{E}\right) \frac{\mu}{\kappa+1} \cdot \sqrt{2} \cdot \sqrt{\frac{\pi}{l}}
\end{aligned}
$$

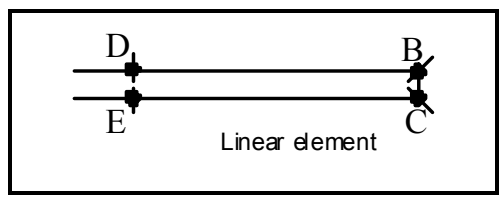

Figure 1: $\quad$ Crack tip at points B and C.

\section{Numerical examples}

A rectangular plate containing a single horizontal edge crack shown in Figure 2 used a mesh with 48 linear elements plus 8 elements on each crack surface (64 B.E.). The crack length is a, the plate width is $\mathrm{w}$ and the height is $2 \mathrm{~h}$. A uniform traction in the height direction was symmetrically applied at the ends. Results obtained for the ratio $\mathrm{h} / \mathrm{w}$ equals to 0.5 are shown in Table 1 . Three ratios $\mathrm{a} / \mathrm{w}$ were considered: $0.2,0.4$, and 0.6 . Stress intensity factor were obtained with eq. (11).

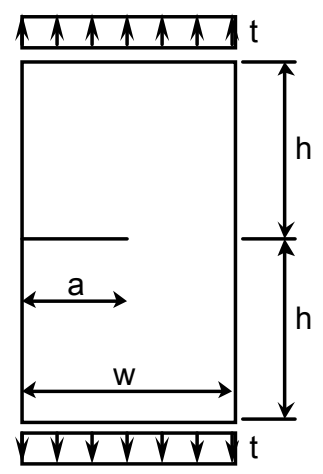

Figure 2: $\quad$ Single edge crack. 
Table 1: $\quad$ Single edge crack with 64 linear elements: $K_{I} /(t \sqrt{\pi a})$.

\begin{tabular}{|c|c|c|c|c|}
\hline $\mathrm{a} / \mathrm{w}$ & {$[6]$} & {$[1]$} & {$[3]$} & Obtained \\
\hline 0.2 & 1.488 & 1.566 & 1.501 & 1.642 \\
\hline 0.4 & 2.324 & 2.230 & 2.368 & 2.328 \\
\hline 0.6 & 4.152 & 4.580 & 4.254 & 4.209 \\
\hline
\end{tabular}

A rectangular plate containing a central slant crack shown in Figure 3 had a mesh with 48 linear elements plus 12 elements on each crack surface (72 B.E.). Crack length is $2 \mathrm{a}$, the plate width is $2 \mathrm{w}$ and the height is $2 \mathrm{~h}$. A uniform traction in the height direction was symmetrically applied at the ends. Results obtained for $\mathrm{h} / \mathrm{w}$ ratio equal to 2 are shown in Table 2 for $\mathrm{K}_{\mathrm{I}}$ and in Table 3 for $\mathrm{K}_{\mathrm{II}}$. Three $\mathrm{a} / \mathrm{w}$ ratios were considered: $0.2,0.4$, and 0.6 . Stress intensity factors were obtained with eq. (11) and eq. (12).

A rectangular plate containing an internal kinked crack shown in Figure 4 had a mesh with 60 linear elements plus 10 elements on each horizontal crack surface and 8 elements on each inclined crack surface. One of the segments of the crack is horizontal with length $b$ while the other segment makes an angle of 45 degrees with the horizontal and has length a; the horizontal projection of the total crack is given by $2 c=b+a(\sqrt{ } 2) / 2$. The kink of the crack is at the center of the plate, the plate width is $2 \mathrm{w}$ and the height is $2 \mathrm{~h}$. Three $\mathrm{a} / \mathrm{b}$ ratios were considered: $0.2,0.4$ and 0.6. The results obtained for $\mathrm{b} / \mathrm{w}$ equal to 0.1 are shown in Tables 4 to 7 . Stress intensity factors were obtained with eq. (11) and eq. (12).

Table 2: $\quad$ Central slant crack with 72 linear elements: $K_{I} /(t \sqrt{\pi a})$.

\begin{tabular}{|c|c|c|c|c|}
\hline $\mathrm{a} / \mathrm{w}$ & {$[7]$} & {$[1]$} & {$[3]$} & Obtained \\
\hline 0.2 & 0.518 & 0.531 & 0.513 & 0.512 \\
\hline 0.4 & 0.572 & 0.588 & 0.567 & 0.566 \\
\hline 0.6 & 0.661 & 0.686 & 0.660 & 0.658 \\
\hline
\end{tabular}

Table 3: Central slant crack with 72 linear elements: $K_{I I} /(t \sqrt{\pi a})$.

\begin{tabular}{|c|c|c|c|c|}
\hline $\mathrm{a} / \mathrm{w}$ & {$[7]$} & {$[1]$} & {$[3]$} & Obtained \\
\hline 0.2 & 0.507 & 0.519 & 0.502 & 0.501 \\
\hline 0.4 & 0.529 & 0.541 & 0.523 & 0.522 \\
\hline 0.6 & 0.567 & 0.579 & 0.561 & 0.557 \\
\hline
\end{tabular}




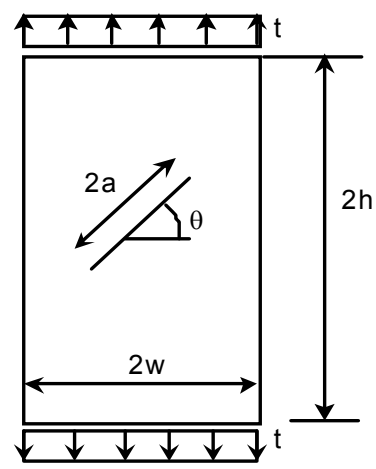

Figure 3: Central slant crack.

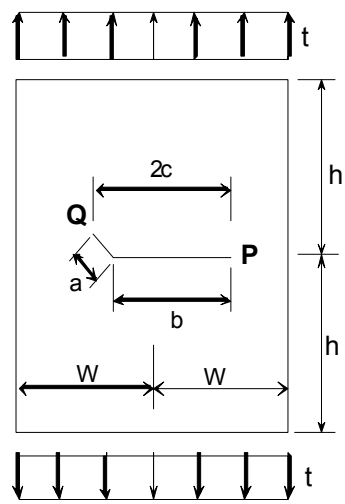

Figure 4: $\quad$ Central kinked crack.

Table 4: $\quad$ Kinked crack with 96 linear elements, results at P: $K_{I} /(t \sqrt{\pi a})$.

\begin{tabular}{|c|c|c|c|c|}
\hline $\mathrm{a} / \mathrm{w}$ & {$[7]$} & {$[1]$} & {$[3]$} & Obtained \\
\hline 0.2 & 0.995 & 1.021 & 0.988 & 0.988 \\
\hline 0.4 & 0.990 & 1.018 & 0.985 & 0.984 \\
\hline 0.6 & 0.986 & 1.017 & 0.983 & 0.982 \\
\hline
\end{tabular}

The obtained values were close to the literature. The adopted meshes were similar to those suggested in [1]. Conformal interpolations were used along the straight lines shown in Figures 2 to 4 . Double nodes were introduced at corners and at crack tips. Collocation points for displacement BIE were positioned at element nodes in conformal interpolations and shifted to the interior of element 
for double nodes at corners and crack tips. Collocation points for traction BIE were always positioned inside the elements. Internal positions for collocations point were studied in [8] for the present strategy and the best position $( \pm 0.67)$, which was adopted in this study, was equal to that obtained in [2] and used in [3]. By the way, when a/w is equal to 0.2 , values for $K_{I}$ in Table 1 and in Table 6 as well as for $\mathrm{K}_{\mathrm{II}}$ in Table 7 could be improved if $\xi^{\prime}$ could be equal to \pm 0.75 .

Table 5: $\quad$ Kinked crack with 96 linear elements, results at $\mathrm{P}: K_{I I} /(t \sqrt{\pi a})$.

\begin{tabular}{|c|c|c|c|c|}
\hline $\mathrm{a} / \mathrm{w}$ & {$[7]$} & {$[1]$} & {$[3]$} & Obtained \\
\hline 0.2 & 0.028 & 0.030 & 0.029 & 0.028 \\
\hline 0.4 & 0.033 & 0.036 & 0.035 & 0.035 \\
\hline 0.6 & 0.030 & 0.032 & 0.032 & 0.032 \\
\hline
\end{tabular}

Table 6: $\quad$ Kinked crack with 96 linear elements, results at Q: $K_{I} /(t \sqrt{\pi a})$.

\begin{tabular}{|c|c|c|c|c|}
\hline $\mathrm{a} / \mathrm{w}$ & {$[7]$} & {$[1]$} & {$[3]$} & Obtained \\
\hline 0.2 & 0.598 & 0.634 & 0.636 & 0.630 \\
\hline 0.4 & 0.574 & 0.603 & 0.606 & 0.603 \\
\hline 0.6 & 0.568 & 0.595 & 0.600 & 0.595 \\
\hline
\end{tabular}

Table 7: $\quad$ Kinked crack with 96 linear elements, results at Q: $K_{I I} /(t \sqrt{\pi a})$.

\begin{tabular}{|c|c|c|c|c|}
\hline $\mathrm{a} / \mathrm{w}$ & {$[7]$} & {$[1]$} & {$[3]$} & Obtained \\
\hline 0.2 & 0.557 & 0.589 & 0.590 & 0.591 \\
\hline 0.4 & 0.607 & 0.637 & 0.639 & 0.635 \\
\hline 0.6 & 0.627 & 0.659 & 0.661 & 0.655 \\
\hline
\end{tabular}

\section{Conclusions}

The present implementation for DBEM considered conformal interpolation along crack surfaces using same expressions for shape functions of conformal and nonconformal interpolations with nodal parameter always fixed at the ends of elements. Internal positions for collocations points were used to non-conformal interpolations for displacement BIE or to all interpolation types for traction BIE. This implementation can be understood as a current code for displacement BIE 
improved with the traction BIE formulation to treat linear fracture mechanics problems, i.e. no special care was necessary in the implementation to introduce the displacement BIE to treat linear fracture mechanics.

Thus, the effect of the collocation point position is only related to the collocation point for traction BIE. The present results were similar to those obtained with internal points for both BIEs in [3] as well as to those obtained with quadratic elements using non-conformal interpolations along crack surfaces in [1].

The stress intensity factors were obtained using near-tip displacement extrapolation and it is important to note that better values for them could be obtained with J-integral technique [1], which is a post processing technique that could be applied to the present formulation but was not used in this paper.

This study strongly suggests the use of the TDO due to the simplifications to treat the singularity (the treatment of the improper integrals only used the first order finite part). Furthermore, the use of derivatives of the adopted shape function for displacement without using other interpolation for TDO was an interesting alternative. It is important to note that constant values were obtained as derivatives of the linear shape function and the results were not degraded.

\section{Acknowledgement}

The author is grateful to $\mathrm{CNPq}$ for support for the development of the present research.

\section{References}

[1] Portela, A, Aliabadi, MH, Rooke, DP, The dual boundary element method: Effective implementation for crack problems, International Journal of Numerical Methods in Engineering, 33, 1269-1287, 1992.

[2] Almeida, L.P.C.P.F., Palermo Jr., L, On the Implementation of the Two Dimensional Dual Boundary Element Method for Crack Problems, "5th International Conference on Boundary Elements Techniques" Lisboa, Portugal, 2004.

[3] Palermo Jr., L., Almeida, L.P.C.P.F. and Gonçalves, P.C., The Use of the Tangential Differential Operator in the Dual Boundary Element Equation, Structural Durability \& Health Monitoring, vol.2, no.2, pp.123-130, Tech Science Press, 2006.

[4] Bonnet, M. Boundary Integral Equation Methods for Solids and Fluids, John Wiley \& Sons Ltd, 1999.

[5] Kane, J. H., Boundary Element Analysis in Engineering Mechanics. Prentice Hall, 1994.

[6] Civelek, M. B., Erdogan, F., 1982, Crack problems for a rectangular sheet and an infinite strip, International Journal of Fracture, vol.19, pp.139-159.

[7] Murakami, Y., 1987, Stress Intensity Factors Handbook, Pergamon Press, Oxford. 
84 Boundary Elements and Other Mesh Reduction Methods XXXII

[8] Palermo Jr., L., Gonçalves, P. C., Figueiredo, L. G., The collocation point position in the dual boundary element equation with the tangential differential operator, 19th International Congress of Mechanical Engineering, Brasília, Brasil, 2007. 